\title{
Clinical application of tumor volume in advanced nasopharyngeal carcinoma to predict outcome
}

\author{
Ching-Chih Lee ${ }^{1,5}$, Tze-Ta Huang ${ }^{2}$, Moon-Sing Lee ${ }^{3,5}$, Shih-Hsuan Hsiao ${ }^{1}$, Hon-Yi Lin ${ }^{3,5}$, Yu-Chieh Su ${ }^{4,5}$, \\ Feng-Chun $\mathrm{Hsu}^{3}$, Shih-Kai Hung ${ }^{3,5^{*}}$
}

\begin{abstract}
Background: Current staging systems have limited ability to adjust optimal therapy in advanced nasopharyngeal carcinoma (NPC). This study aimed to delineate the correlation between tumor volume, treatment outcome and chemotherapy cycles in advanced NPC.

Methods: A retrospective review of 110 patients with stage III-IV NPC was performed. All patients were treated first with neoadjuvant chemotherapy, then concurrent chemoradiation, and followed by adjuvant chemotherapy as being the definitive therapy. Gross tumor volume of primary tumor plus retropharyngeal nodes (GTVprn) was calculated to be an index of treatment outcome.

Results: GTVprn had a close relationship with survival and recurrence in advanced NPC. Large GTVprn ( $\geqq 13$ ml) was associated with a significantly poorer local control, lower distant metastasis-free rate, and poorer survival. In patients with GTVprn $\geqq 13 \mathrm{ml}$, overall survival was better after $\geqq 4$ cycles of chemotherapy than after less than 4 cycles.
\end{abstract}

Conclusions: The incorporation of GTVprn can provide more information to adjust treatment strategy.

\section{Background}

Nasopharyngeal carcinoma (NPC) is a unique malignant head and neck cancer with a specific behavior. It is rarely reported in the West but occurs at high frequency in Southern China, Hong Kong, Taiwan, Singapore, and Malaysia [1]. Radiotherapy has long been the standard treatment for patients with NPC because of its anatomic location and relative radiosensitivity. Based on the American Joint Committee on Cancer (AJCC) staging system in 1997, and the 5-year overall survival rates for tumor stages I, II, III, and IV were $95-70 \%, 83-65 \%$, 76$54 \%$, and $56-29 \%$, respectively [2-6]. Although NPC is markedly radiosensitive, a high rate of treatment failure is observed in patients with advanced NPC especially distant failure. Combination chemotherapy plus radiotherapy has been widely accepted as the treatment modality for advanced NPC; however, treatment strategies for this disease have yet to be optimized [7-9].

\footnotetext{
* Correspondence: oncology158@yahoo.com.tw
${ }^{3}$ Department of Radiation Oncology, Buddhist Dalin Tzu Chi General

* Correspondence: oncology158@yahoo.com.tw
${ }^{3}$ Department of Radiation Oncology, Buddhist Dalin Tzu Chi General Hospital, Chiayi, Taiwan 62247
} Hospital, Chiay, Taiwan 62247

The accurate prediction of prognosis and failure is crucial for optimizing therapy. In general, the 1997 AJCC staging system is the most widely used staging system for NPC [2]. However, the current TNM staging system is based on anatomic location and cranial nerve involvement that still has limitations. In addition to well established prognostic factors such as tumor stage, histopathologic type, and cranial nerve involvement, primary tumor volume has been recognized as a promising prognostic indicator in the treatment of NPC [10-13]. Since use of tumor volume could improve the ability of the current staging system to predict outcome, this study aimed to delineate the correlation between tumor volume, treatment outcome and chemotherapy cycles in NPC treated with multimodality therapy.

\section{Methods \\ Patients}

For this retrospective analysis, the treatment records of 142 patients with stage III-IV NPC (AJCC system) [2] from August 2000 to February 2007 in an institution were reviewed. Thirty-two patients were excluded because of distant metastasis present at initial diagnosis, 
loss to follow up, performance status $>2$, or a synchronous second primary tumor. The histological diagnosis of NPC was made by experienced pathologists. None of the patients received prior treatment for their cancer. All patients were informed about the treatment of neoadjuvant chemotherapy then concurrent chemoradiation (CCRT) and followed by adjuvant chemotherapy as being the definitive therapy for advanced disease, including the potential benefits and possible side effects. All patients were treated by multidisciplinary teams including a head and neck surgery team, radiation oncologists, medical oncologists and dieticians.

\section{Tumor volume measurement}

All patients in this study underwent pre-treatment, contrast-enhanced CT scan that were done along the axial scan plain parallel to the infraorbital-meatal line extending from the skull base to the top of manubrium using 3-5 mm sections. Direct coronal scans also were taken to provide auxiliary information. One hundred millimeters of contrast medium were administrated with an injection rate between $1 \mathrm{~mL}$ per second and $2.5 \mathrm{~mL}$ per second after an initial 5-mL dose. Gross tumor volume of primary tumor plus retropharyngeal nodes (GTVprn) was included (depending on the imaging system) in the tumor volume measurement. First, manual tracing was performed using a graphic user interface, and area inside the outline was automatically labeled and calculated. The volume was calculated by multiplying the sum of all areas by the slice thickness (image reconstruction interval). All images were evaluated by two clinicians at least. A radiologist who specialized in head and neck cancer participated when the outline of tumor margin was unclear.

\section{Radiotherapy}

An intensity-modulated radiation therapy (IMRT) technique and inverse planning system (PLATO, Nucleotron Inc, Veenendaal, Netherlands) were used for treatment delivery. The radiation field encompassed the primary tumor bed and neck lymph nodes. The prescribed dose of external beam treatment was 72 Gy to the gross tumor and positive neck nodes, 63 Gy to the clinical target volume, and 50-60 Gy to the clinically negative neck. Doses were delivered at $1.8 \mathrm{~Gy} /$ day for five consecutive days by a linear accelerator with patients lying supine with a mask. After 1-2 weeks of completing the external beam radiotherapy, an intracavitary brachytherapy boost (3.5 Gy to the submucosa $0.5 \mathrm{~cm}$ in 3 fractions) was prescribed if residual tumor was suspected. The intracavitary brachytherapy boost was conducted using highdose-rate (HDR) afterloader unit (microSelectron-HDR, Nucleotron Inc, Veenendaal, Netherlands) containing an iridium-192 source.

\section{Chemotherapy}

All consenting patients were eligible for chemotherapy if they met the following criteria: ECOG performance status $\leq 2$, serum creatinine level $<1.5 \mathrm{mg} / \mathrm{dL}$, absolute neutrophil count $\geq 2000$ cells $/ \mu \mathrm{L}$, and platelet count $>10,000 / \mu \mathrm{L}$. The chemotherapy protocol consisted of 6 monthly cycles of cisplatin $\left(100 \mathrm{mg} / \mathrm{m}^{2} /\right.$ day $)$ on Day 1 followed by 5 -FU $\left(1000 \mathrm{mg} / \mathrm{m}^{2} /\right.$ day $)$ continuously infused for 5 consecutive days, in the presence of adequate hydration and anti-emetic drugs.

\section{Dose modification}

Toxicity was evaluated using the common toxicity criteria of the National Cancer Institute. Both cisplatin and 5 -FU were withheld if the absolute neutrophil count was $<1500$ cells $/ \mu \mathrm{L}$ or if the platelet count was $<75,000$ cells/ $\mu \mathrm{L}$. Both agents were given at $70 \%$ of the initial dose if the neutrophil count was $1500-2000$ cells $/ \mu \mathrm{L}$ or if the platelet count was 75,000-100,000 cells/ $\mu \mathrm{L}$. Radiotherapy was withheld only if the neutrophil count was $<1000$ cells $/ \mu \mathrm{L}$ or if the platelet count was $<50,000$ cells $/ \mu \mathrm{L}$. For grades 3 and 4 oropharyngeal mucositis or diarrhea, 5-FU was withheld until the symptoms improved. It was then restarted at $70 \%$ of the initial dose. For grades 3 and 4 renal toxicity, cisplatin was withheld until the creatinine was $<1.5 \mathrm{mg} / \mathrm{dL}$. It was administered at $70 \%$ of the initial dose thereafter.

\section{Patient evaluation}

Survival was calculated from the date of diagnosis to the most recent follow-up or to the date of recurrence or death. The pattern of failure was defined according to the first site of failure: local failure defined as recurrence of the primary tumor or metastasis to regional lymph nodes; and distant failure indicating metastasis to any site beyond the primary tumor and regional lymph nodes. After recurrence or metastasis, patients were given salvage therapy as determined by their physicians.

\section{Statistical analysis}

Different groups were compared with respect to baseline characteristics, with the $t$-test used for continuous variables and the chi-square test for categorical variables. The Kaplan-Meier method was used for survival analysis [14]. The difference between survival curves was determined using the log-rank test [15]. Multivariate analysis to identify significant prognostic factors was accomplished by Cox regression model. The receiver operating characteristic (ROC) curve analysis was applied to evaluate different cut-off point of tumor size in order to find the appropriate size for clinical application. SPSS 12.0 software (SPSS Inc, Chicago, IL, USA) was used for analysis of all data. Statistical significance was accepted as a $p$ value of less than 0.05 . 


\section{Results}

Patient baseline characteristics are presented in Table 1. Median patient follow-up at the commencement of the analysis was 38 months (range 4-107). All patients received the radiation. After extern beam radiotherapy, 13 of the 110 patients received an intracavitary brachytherapy boost $(3.5$ Gy to the submucosa $0.5 \mathrm{~cm}$ in 3 fractions) because residual tumor was suspected. Median cumulative radiation dose delivered over the study duration was $7200 \mathrm{cGy}$ (range 6120-8250 cGy). For the rate of compliance with chemotherapy treatment, the patient received $\geqq 4$ and $<4$ cycles of chemotherapy were $42 \%$ and $58 \%$, respectively. During follow up, 7 patients had recurrence at the primary site, 23 patients had distant metastasis, and 5 patients combined recurrence with metastasis. The metastasis sites were the bone, lung, and liver. The 3-year overall survival, disease-free survival, local control, and distant metastasis-free rates in all patients were $67 \%, 66 \%, 80 \%$, and $66 \%$, respectively.

Table 1 Patient Characteristics

\begin{tabular}{|c|c|}
\hline Patient characteristic & No. of patients (\%) \\
\hline All & $110(100)$ \\
\hline \multicolumn{2}{|l|}{$\overline{\text { Age }}$} \\
\hline Median & 52 \\
\hline Range & $26-73$ \\
\hline \multicolumn{2}{|l|}{ Gender } \\
\hline Male & $71(64.5)$ \\
\hline Female & $39(35.5)$ \\
\hline \multicolumn{2}{|l|}{ Histology } \\
\hline WHO type I & $3(2.7)$ \\
\hline WHO type ॥ & $84(76.4)$ \\
\hline WHO type III & $23(20.9)$ \\
\hline \multicolumn{2}{|l|}{ Performance status (ECOG) } \\
\hline 0 & $103(93.6)$ \\
\hline 1 & $4(3.6)$ \\
\hline 2 & $3(2.7)$ \\
\hline \multicolumn{2}{|l|}{ AJCC 1997 T stage } \\
\hline 1 & $20(18.2)$ \\
\hline 2 & $35(31.8)$ \\
\hline 3 & $23(20.9)$ \\
\hline 4 & $32(29.1)$ \\
\hline \multicolumn{2}{|l|}{ AJCC 1997 N stage } \\
\hline 0 & $2(1.8)$ \\
\hline 1 & $3(2.7)$ \\
\hline 2 & $75(68.2)$ \\
\hline 3 & $30(27.3)$ \\
\hline \multicolumn{2}{|l|}{ AJCC 1997 Stage group } \\
\hline III & $61(55.5)$ \\
\hline IV & $49(44.5)$ \\
\hline
\end{tabular}

WHO, World Health Organization; ECOG, Eastern Cooperative Oncology Group; AJCC, American Joint Committee on Cancer.
Table 2 T Stage and GTVprn

\begin{tabular}{lcccc}
\hline & \multicolumn{2}{c}{ GTVprn $(\mathbf{m l})$} & \multicolumn{2}{c}{ Patients (\%) } \\
\cline { 2 - 5 } T stage & Median & Range & GTVprn $<\mathbf{1 3} \mathbf{~ c m}$ & GTVprn $\geq \mathbf{1 3} \mathbf{~ c m}$ \\
\hline T1 $(\mathrm{n}=20)$ & 4.60 & $1.3-11.4$ & $20(100)$ & $0(0)$ \\
T2 $(\mathrm{n}=35)$ & 15.3 & $2.3-37.2$ & $17(49)$ & $18(51)$ \\
T3 $(\mathrm{n}=23)$ & 21.3 & $3.6-130.7$ & $14(61)$ & $9(39)$ \\
T4 $(\mathrm{n}=32)$ & 31.9 & $6.7-166.6$ & $12(38)$ & $20(62)$ \\
\hline
\end{tabular}

GTVprn, gross tumor volume of primary tumor plus retropharyngeal nodes

Table 2 shows the $\mathrm{T}$ stage distribution and GTVprn for each stage. The median GTVprn of stage III to IV were 14.1 and $25.5 \mathrm{ml}$ and corresponding ranges were 1.3-130.7 and 2.2-166.6 ml. The optimal GTVprn to find the appropriate size for clinical application was 13 ml. For GTVprn analysis, the 3-year overall survival, disease-free survival, local control, and distant metastasisfree rate in subgroups with GTVprn $<13 \mathrm{ml}$ and $\geqq 13$ $\mathrm{ml}$ were $92 \% / 54 \%, 86 \% / 25 \%, 95 \% / 63 \%$, and $88 \% / 51 \%$, respectively. Large GTVprn ( $\geqq 13 \mathrm{ml}$ ) was associated with a significantly poorer local control, lower distant metastasis-free rate, and poorer survival (Figure 1). Besides, larger GTVprn ( $\geqq 18 \mathrm{ml}$ ) was found to be correlation with $\mathrm{N}$ stage which was a significant prognostic factor on univariate analysis in distant metastasis free rate. Analysis of the subgroup with GTVprn $\geqq 13 \mathrm{ml}$ revealed better overall survival after $\geqq 4$ cycles of chemotherapy than after less than 4 cycles (Figure 2).

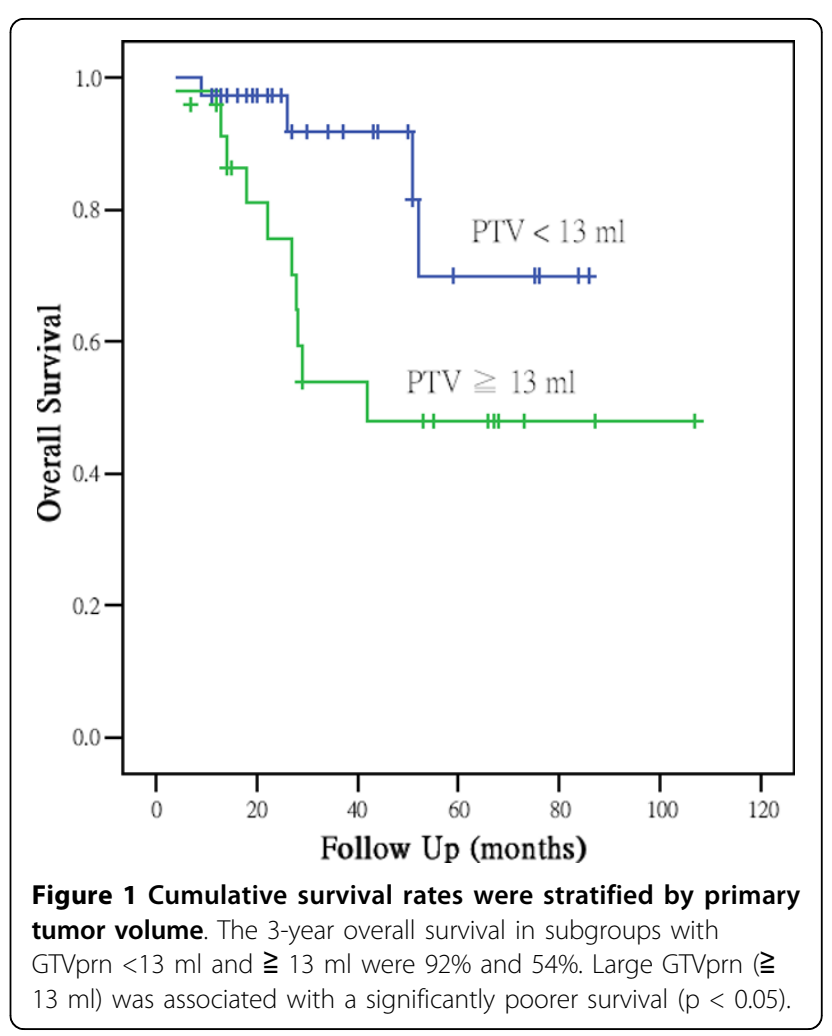




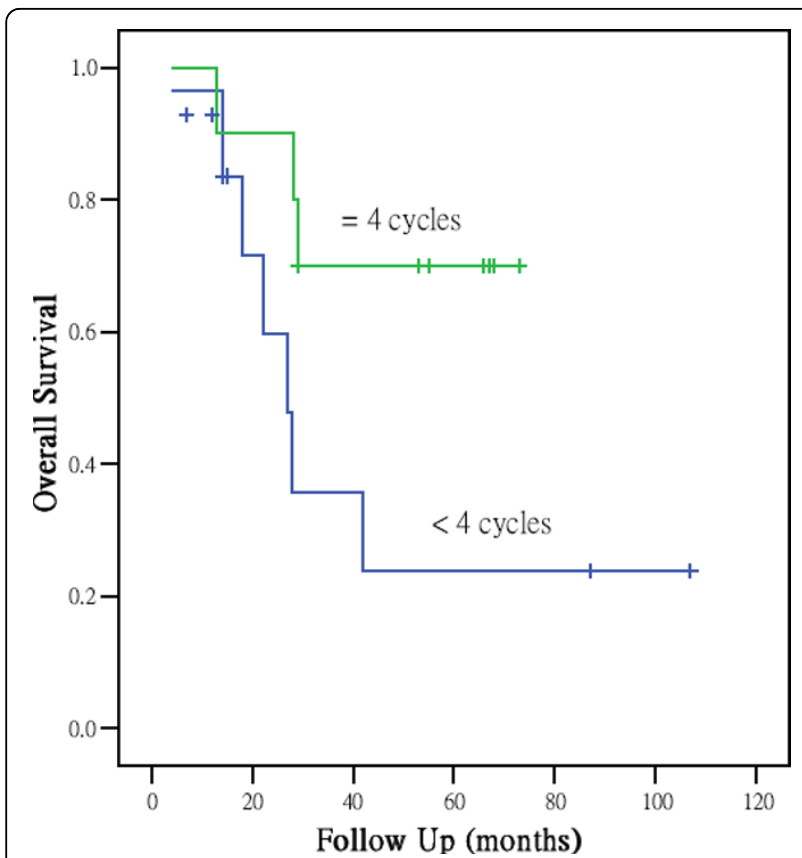

Figure 2 Analysis of the subgroup with GTVprn $\geqq 13 \mathrm{ml}$ revealed better overall survival after $\geqq 4$ cycles of chemotherapy than after less than 4 cycles. $(p<0.05)$.

Patients' 3-year overall survival, disease-free survival, local control, and distant metastasis-free rates were $70 \% / 24 \%, 63 \% / 30 \%, 78 \% / 76 \%$, and $65 \% / 42 \%$, respectively. A Cox proportional hazard regression model was constructed to calculate the relative risks and confidence intervals for different prognostic factors after controlling for age and gender. The results are summarized in Table 3. Only GTVprn was found to be an independent factor. Survival analysis demonstrated a significant difference in overall survival with larger tumor volume (risk ratio, 2.92; $\mathrm{p}=0.02$ ).

\section{Discussion}

The accurate prediction of prognosis and failure is crucial for optimizing therapy. We addressed the question of whether the AJCC staging system is adequate for predicting the prognosis of patients with NPC. NPC is often highly infiltrated and heterogeneous in all disease stages. Recently, tumor volume has been evaluated as a predictor because of the relationship of large volume with adverse biologic factors, including clonogen number, hypoxia, and radioresistance [11]. Several studies had demonstrated primary tumor volume could improve the current staging system [10-13]. Chua et al. found that primary tumor volume is an independent prognostic factor of local control and apparently more predictive than Ho's T stage classification [12]. Chen et al. also demonstrated that primary tumor volume predicted survival of patients with NPC with more accuracy than the AJCC staging system [10]. This indicates the limitation of the current TNM staging system based on anatomic location in separation of tumor bulk. Base on previous study, primary tumor volume was found to be a significant prognosis factor for treatment outcome and significantly correlated with $\mathrm{T}$ stage [13]. In the current study, additional testing was performed in an attempt to define the critical volume in the advanced NPC. We used a cut off value of $13 \mathrm{ml}$ to divide patients into different prognostic groups. GTVprn $\geqq 13 \mathrm{ml}$ was associated with a significantly poorer local control, lower distant metastasis-free rate, and poorer survival. Since most of our patients had stage N2 and N3 tumors, we tried to determine whether GTVprn was correlated with $\mathrm{N}$ stage. However, GTVprn would be increased to 18 $\mathrm{ml}$ which could be correlated with $\mathrm{N}$ stage. Because the cut-off value of $13 \mathrm{ml}$ could be used to predict and adjust treatment strategy. These results led us to hypothesize that GTVprn can refine the staging system and to speculate that micrometastases may sometimes occur before neck lymph node involvement is apparent.

Distant metastasis is an important concern that can influence survival. The reported frequency of distant metastases in patients with locally advanced NPC was greater than $30 \%$ with radiotherapy alone [7]. An autopsy series had shown a high rate of distant metastases (38-87\%) involving virtually every organ [16].

Table 3 Cox Proportional Hazard Model Analysis

\begin{tabular}{|c|c|c|c|c|c|c|c|c|c|c|}
\hline Variables & $\begin{array}{l}\text { Overall } \\
\text { survival } \\
(\mathrm{RR}, 95 \% \mathrm{Cl})\end{array}$ & $p$ & $\begin{array}{l}\text { Disease-specific } \\
\text { survival } \\
(\mathrm{RR}, 95 \% \mathrm{Cl}))\end{array}$ & $p$ & $\begin{array}{l}\text { Disease-free } \\
\text { survival } \\
\text { (RR, 95\% Cl) }\end{array}$ & $p$ & $\begin{array}{l}\text { Loco-regional } \\
\text { control } \\
\text { (RR, 95\% Cl) }\end{array}$ & $p$ & $\begin{array}{l}\text { Distant } \\
\text { metastasis- } \\
\text { free survival } \\
(\mathrm{RR}, 95 \% \mathrm{Cl})\end{array}$ & $p$ \\
\hline T1-2 vs. T3-4 & $1.29,0.56-2.98$ & 0.56 & $1.42,0.41-4.86$ & 0.58 & $0.85,0.34-2.16$ & 0.73 & $1.44,0.27-7.64$ & 0.67 & $1.00,0.36-2.81$ & 1.00 \\
\hline N0-2 vs. N3 & $2.47,0.48-12.71$ & 0.28 & $1.86,0.15-23.26$ & 0.63 & $2.85,0.53-15.18$ & 0.22 & $39.06,1.55-983.27$ & 0.03 & $2.77,0.54-14.07$ & 0.22 \\
\hline $\begin{array}{l}\text { Cranial nerve } \\
\text { involvement }\end{array}$ & $0.71,0.26-1.95$ & 0.51 & $0.73,0.17-3.13$ & 0.67 & $0.65,0.18-2.28$ & 0.50 & $0.11,0.006-2.10$ & 0.14 & $0.95,0.26-3.46$ & 0.94 \\
\hline $\begin{array}{l}\text { Supraclavicular } \\
\text { nodes }\end{array}$ & $0.74,0.15-3.74$ & 0.71 & $0.78,0.06-9.59$ & 0.85 & $0.45,0.08-2.50$ & 0.36 & $0.02,0.001-0.87$ & 0.04 & $0.68,0.13-3.43$ & 0.64 \\
\hline GTVprn (13 ml) & $2.92,1.22-6.98$ & 0.02 & $4.10,1.06-15.97$ & 0.04 & $4.81,1.73-13.36$ & $<0.01$ & $16.83,1.48-190.78$ & 0.02 & $2.50,0.84-7.43$ & 0.10 \\
\hline
\end{tabular}

$\mathrm{RR}$, risk ratio; $95 \% \mathrm{Cl}, 95 \%$ confidence interval; GTVprn, gross tumor volume of primary tumor plus retropharyngeal nodes 
Systemic chemotherapy was included in this investigation in an attempt to reduce the incidence of distant metastasis. In this study, failure pattern analysis revealed that the number of distant metastasis sites was greater than the number of local recurrence sites. The 3-year distant metastasis-free rate of $66 \%$ demonstrated that distant metastasis was still the main challenge in advanced NPC. In addition to delineate the cut off volume into different prognostic groups, we also analyzed the prognosis of subgroups with different conditions. We found that the subgroup with GTVprn $\geqq 13$ $\mathrm{ml}$ revealed longer survival after $\geqq 4$ cycles of chemotherapy than after less than 4 cycles. These results may hint the need for adequate systemic cycle regimes to eradicate micrometastases and improve survival. However, it is important to note that half of patients required treatment modification during chemotherapy or refused further treatment. More effective and safer drugs should be considered for integration into multimodal treatment strategies.

Although incorporation of primary tumor volume could improve the accuracy of the current staging system, it still has some problems. One consideration is how tumor volume is quantitatively determined. Clinical care requires a classification system that reflects the current state of scientific knowledge and can guide clinical decision-making. NPC tumor volume measurement is a complicated procedure and the results may be affected by imaging modalities (CT or MR imaging), measuring protocols, and measurement techniques [17]. Rasch et al. indicated that MRI-derived tumor volume is smaller and has less interobserver variation than CT-derived tumor volume [18]. However, CT cannot be neglected because the geometric accuracy of the patient contour is poorer in MRI and lacks electron density information [10,19]. In addition, measurement of tumor volume is still time-consuming, labourintensive, and not widely available. Tumor volume definition and measuring protocols should be standardized in clinical practice. Lee et al. had tried to use simple measurement to evaluate of primary tumor volume and seemed feasible [13]. Otherwise, although large tumor volume was more commonly observed in the higher stages, there were large variation tumor volume and much overlapping among different stages [10-12]. Its means similar values of tumor volume could have different treatment response. Other factors such as tumor extension, intrinsic resistance, or hypoxia should be considered for integration into the staging system.

\section{Conclusions}

Since this is a retrospective study, a number of factors in terms of patient and tumor characteristics could not be controlled and may have biased the results. Nevertheless, it appears that incorporation of tumor volume can further refine the staging system and adjust treatment strategy. For patients with large GTVprn ( $\mathrm{ml}$ ), the use of more effective and safer drugs with adequate systemic cycles is suggested.

\section{Author details}

${ }^{1}$ Department of Otolaryngology, Buddhist Dalin Tzu Chi General Hospital, Chiayi, Taiwan 62247. 'Department of Oral and Maxillofacial Surgery, Buddhist Dalin Tzu Chi General Hospital, Chiayi, Taiwan 62247. ${ }^{3}$ Department of Radiation Oncology, Buddhist Dalin Tzu Chi General Hospital, Chiayi, Taiwan 62247. ${ }^{4}$ Department of Hematological Oncology, Buddhist Dalin Tzu Chi General Hospital, Chiayi, Taiwan 62247. ${ }^{5}$ School of Medicine, Tzu Chi University, Hualian, Taiwan 97061.

\section{Authors' contributions}

LCC and HSK developed the ideas for these experiments, performed much of the work, and drafted the manuscript. LMS, HSH, LHY and SYC designed the study, collected the data and interpreted the data. LCC performed the statistical analysis. All authors read and approved the final manuscript.

\section{Competing interests}

The authors declare that they have no competing interests.

Received: 17 January 2010 Accepted: 11 March 2010

Published: 11 March 2010

\section{References}

1. Liu MT, Hsieh CY, Chang TH, Lin JP, Huang CC, Wang AY: Prognostic factors affecting the outcome of nasopharyngeal carcinoma. Jpn J Clin Oncol 2003, 33:501-8.

2. Greene FL, Page DL, Fleming ID, Fritz A, Balch CM, Haller DG, Morrow M: AJCC Cancer Staging Manual. New York: Springer-Verlag, 6 2002, 47-52.

3. Chua DT, Sham JS, Kwong DL, Choy DT, Au GK, Wu PM: Prognostic value of paranasopharyngeal extension of nasopharyngeal carcinoma. A significant factor in local control and distant metastasis. Cancer 1996, 78:202-10.

4. Cooper JS, Cohen R, Stevens RE: A comparison of staging systems for nasopharyngeal carcinoma. Cancer 1998, 83:213-9.

5. Lee AW, Foo W, Law SC, Poon YF, O SK, Tung SY, Sze WM, Chappell R, Lau WH, Ho JH: Staging of nasopharyngeal carcinoma: from Ho's to the new UICC system. Int J Cancer 1998, 84:179-87.

6. Ozyar E, Yildiz F, Akyol FH, Atahan IL: Comparison of AJCC 1988 and 1997 classifications for nasopharyngeal carcinoma. American Joint Committee on Cancer. Int J Radiat Oncol Biol Phys 1999, 44:1079-87.

7. Al-Sarraf M, LeBlanc M, Giri PG, Fu KK, Cooper J, Vuong T, Forastiere AA, Adams G, Sakr WA, Schuller DE, Ensley JF: Chemoradiotherapy versus radiotherapy in patients with advanced nasopharyngeal cancer: phase III randomized Intergroup study 0099. J Clin Oncol 1998, 16:1310-7.

8. Lin JC, Jan JS, Hsu CY, Liang WM, Jiang RS, Wang WY: Phase III study of concurrent chemoradiotherapy versus radiotherapy alone for advanced nasopharyngeal carcinoma: positive effect on overall and progressionfree survival. J Clin Oncol 2003, 21:631-7.

9. Chan AT, Teo PM, Ngan RK, Leung TW, Lau WH, Zee B, Leung SF, Cheung FY, Yeo W, Yiu HH, Yu KH, Chiu KW, Chan DT, Mok T, Yuen KT, Mo F, Lai M, Kwan WH, Choi P, Johnson PJ: Concurrent chemotherapyradiotherapy compared with radiotherapy alone in locoregionally advanced nasopharyngeal carcinoma: progression-free survival analysis of a phase III randomized trial. J Clin Oncol 2002, 20:2038-44.

10. Chen MK, Chen TH, Liu JP, Chang CC, Chie WC: Better prediction of prognosis for patients with nasopharyngeal carcinoma using primary tumor volume. Cancer 2004, 100:2160-6.

11. Sze WM, Lee AW, Yau TK, Yeung RM, Lau KY, Leung SK, Hung AW, Lee MC, Chappell R, Chan K: Primary tumor volume of nasopharyngeal carcinoma: prognostic significance for local control. Int J Radiat Oncol Biol Phys 2004, 59:21-7.

12. Chua DT, Sham JS, Kwong DL, Tai KS, Wu PM, Lo M, Yung A, Choy D, Leong $L$ : Volumetric analysis of tumor extent in nasopharyngeal carcinoma and correlation with treatment outcome. Int J Radiat Oncol Biol Phys 1997, 39:711-9. 
13. Lee CC, Ho HC, Su YC, Lee MS, Hsiao SH, Hwang JH, Hung SK, Chou P, Lee CC: Bidimensional measurement of nasopharyngeal carcinoma: a simple method to predict outcomes. Clin Otolaryngol 2009, 34:26-33.

14. Kaplan EL, Meier P: Nonparametric estimation for incomplete observation. J Am Stat Assoc 1958, 53:457-81.

15. Mantle N: Evaluation of survival data and two new rank order statistics arising in its consideration. Cancer Chem Rep 1966, 5:163-70.

16. Ahmad A, Stefani S: Distant metastases of nasopharyngeal carcinoma: a study of 256 male patients. I Surg Oncol 1986, 33:194-7.

17. Zhou JY, Chong VF, Khoo JB, Chan KL, Huang J: The relationship between nasopharyngeal carcinoma tumor volume and TNM T-classification: a quantitative analysis. Eur Arch Otorhinolaryngol 2007, 264:169-74.

18. Rasch C, Keus R, Pameijer FA, Koops W, de Ru V, Muller S, Touw A, Bartelink $H$, van Herk M, Lebesque JV: The potential impact of CT-MRI matching on tumor volume delineation in advanced head and neck cancer. Int J Radiat Oncol Biol Phys 1997, 39:841-8.

19. Fraass BA, McShan DL, Diaz RF, Ten Haken RK, Aisen A, Gebarski S, Glazer G, Lichter AS: Integration of magnetic resonance imaging into radiation therapy treatment planning: I. Technical considerations. Int I Radiat Oncol Biol Phys 1987, 13:1897-908.

doi:10.1186/1748-717X-5-20

Cite this article as: Lee et al: Clinical application of tumor volume in advanced nasopharyngeal carcinoma to predict outcome. Radiation Oncology 2010 5:20.

\section{Submit your next manuscript to BioMed Central and take full advantage of:}

- Convenient online submission

- Thorough peer review

- No space constraints or color figure charges

- Immediate publication on acceptance

- Inclusion in PubMed, CAS, Scopus and Google Scholar

- Research which is freely available for redistribution

Submit your manuscript at www.biomedcentral.com/submit 